\title{
Fatores citogenéticos associados a danos reprodutivos em programa universitário de genética comunitária
}

\author{
Cytogenetic factors associated with reproductive damage in community genetics \\ university programs
}

\author{
Vitória Ohana Ramos e Santos ${ }^{1}$, Mônica Jacobina Fonseca Vieira², Lília Maria de Azevedo Moreira ${ }^{3 *}$ \\ ${ }^{1}$ Graduanda em Ciências Biológicas pela UFBA; ${ }^{2}$ Mestre em Medicina e Saúde pela UFBA; \\ ${ }^{3}$ Doutora em Genética pela USP
}

\begin{abstract}
Resumo
Introdução: perdas gestacionais esporádicas são comuns e estima-se que aproximadamente 30 a $50 \%$ de todas as concepções não terminem em um recém-nascido vivo. Patologia materna ou alteração genética de natureza cromossômica, em um dos progenitores, são fatores de risco favoráveis a esses acontecimentos. Outras situações que podem estar relacionadas às alterações cromossômicas são a infertilidade e a ocorrência de mola hidatiforme (neoplasia trofoblástica gestacional), caracterizada por interferência na formação e desenvolvimento do embrião. Embora haja um consenso sobre o caráter multifatorial das perdas gestacionais, incluindo componentes anatômicos, imunológicos, endócrinos, genéticos e ambientais, ainda assim, a causalidade pode permanecer desconhecida. Objetivos: analisar e quantificar achados de dificuldades reprodutivas relacionados a presença de alterações cromossômicas encontrados no serviço de genética realizado pelo programa Genética \& Sociedade, do Instituto de Biologia da Universidade Federal da Bahia. Metodologia: estudo descritivo e analítico, realizado por meio de consulta a fichas de anamneses, preenchidas durante o atendimento de casais, que buscaram o serviço de genética comunitária para aconselhamento genético no período de vinte anos. Resultados: entre os 73 casais selecionados para estudo, $59(80,8 \%)$ relataram abortos recorrentes, $9(12,3 \%)$ tinham histórico de mola hidatiforme e $5(6,8 \%)$ apresentaram infertilidade. Entre as alterações foram verificadas translocações, inversões, além de polimorfismos de regiões heterocromáticas. Conclusões: o presente estudo confirma a importância das alterações cromossômicas na etiologia das dificuldades reprodutivas, justificando a busca pelo aconselhamento genético. Em termos de saúde pública, traz contribuições para o entendimento das condições genéticas da comunidade beneficiada pelo programa Genética \& Sociedade.
\end{abstract}

Palavras-chave: Abortos. Anomalias cromossômicas. Infertilidade. Mola hidatiforme

\begin{abstract}
Introduction: sporadic gestational losses are common, it's estimated that approximately 30 to $50 \%$ of all conceptions don't end in a newborn alive. Maternal pathology or genetic alterations of chromosomal nature in one of the progenitors are risk factors favorable to these events. Other situations that may be related to chromosomal alterations are infertility and the occurrence of hydatidiform mole (gestational trophoblastic neoplasia), characterized by interference in the formation and development of the embryo. Although there is a consensus about the multifactorial nature of gestational losses, including anatomical, immunological, endocrine, genetic and environmental components, however, the causality may remain unknown. Objectives: analyze and quantify the findings of reproductive difficulties related to the presence of chromosomal alterations found in the genetics service performed by the Genética\&Sociedade program of the Biology Institute of the Federal University of Bahia. Methodology: a descriptive and analytical study, carried out by consultation of anamnesis records, made during the care of couples, who sought the community genetic service for genetic counseling in the period of twenty years. Results: among the 73 couples selected, 59(80.8\%) reported recurrent abortions, $9(12.3 \%)$ had a history of hydatidiform mole and 5 (6.8\%) had infertility. Among all the alterations, were found translocations, inversions, and polymorphisms of heterochromatic regions. Conclusions: this study confirms the importance of chromosomal alterations in the etiology of reproductive difficulties, justifying the search for genetic counseling. In terms of public health, it contributes to the understanding of the genetic conditions in the community benefited by the Genética\&Sociedade program.
\end{abstract}

Keywords: Abortion. Chromosomal anomalies. Infertility. Hydatidiform mole.

\section{INTRODUÇÃO}

Partindo do princípio que cerca de 30 a $50 \%$ de todas as concepções não resultam em recém-nascidos vivos (FRANCISCO et al., 2013), o estudo sobre as causas do

Correspondente/Corresponding: * Lília Maria de Azevedo Moreira - Instituto de Biologia, Universidade Federal da Bahia. Rua Barão de Jeremoabo, 668-Campus Ondina - CEP:40170-115-Salvador, Ba - Tel.: (71) 3283-6538-Email: lazevedo@ufba.br. insucesso reprodutivo constitui um tema relevante em termos de saúde pública. A perda gestacional a partir de três vezes sucessivas é tida como recorrente e apresenta-se com prevalência de 0,5 a 3\% na população (FRANCISCO et al., 2013).

A etiologia da perda gestacional é multifatorial e entre as associações conhecidas estão anormalidades endócrinas, fatores anatômicos, imunológicos, genéticos, distúrbios trombolíticos herdados e agentes infecciosos 
(MARQUI, 2018). Em algumas situações a causalidade não é identificada sendo considerada idiopática. Entre os fatores genéticos de influência estão também os rearranjos cromossômicos balanceados nos progenitores e os cariótipos embrionários anormais. Em relação ao cariótipo dos progenitores, estima-se que em aproximadamente $3 \%$ a $5 \%$ dos casais, com histórico de abortos sucessivos, um dos parceiros apresente alguma anomalia citogenética, o que por sua vez, é cerca de cinco a seis vezes maior do que o observado na população adulta em geral (DIBI; PFEIL; PASKULIN, 2009), tornando relevante conhecer o cariótipo do casal nos casos de abortos recorrentes.

Entre as anormalidades cromossômicas mais comuns em humanos estão as translocações, que consistem na troca de segmentos entre cromossomos não homólogos. Dentre estas, estão as translocações recíprocas, presentes em 1 a cada 500 recém-nascidos, para os quais as chances de conceber, na fase adulta, um embrião cromossomicamente anormal, varia de $20 \%$ a $80 \%$ a depender da translocação (EVSIKOV; CIESLAK; VERLINSK, 2000).

Existe também relação entre o período da perda e a presença de anomalia cromossômica: quanto mais precoce o momento do aborto, mais provável a associação. Estima-se que a presença de anomalias citogenéticas seja de $90 \%$ nas perdas até a quinta semana e de $50 \%$ entre a sexta e a nona semana gestacional (FRANCISCO et al., 2013). Nesses casos, é relevante destacar que entre as alterações citogenéticas encontradas, há maior prevalência das anomalias numéricas e que, em boa parcela dos casos, tratam-se de situações novas não havendo alteração nos cariótipos parentais.

Outro possível motivo da interrupção da gestação é a ocorrência de mola hidatiforme (MH), caracterizada como uma doença trofoblástica gestacional, na qual, a gravidez anormal tem desenvolvimento inadequado do ovo, apresentando óbito do feto ou não desenvolvimento do mesmo, com transformação da placenta em numerosas vesículas preenchidas por líquido. A mola hidatiforme pode ser completa (MHC), com ausência total de elementos fetais, ou parcial (MHP), com presença de feto, havendo distinção entre a origem das mesmas. A MHP é ocasionada pela adição de um conjunto extra de cromossomos de origem paterna devido a fertilização de oócito normal por espermatozoide não haploide, ou por ocorrência de dispermia (caracterizada por dupla fecundação). Na MHC há dissomia uniparental paterna, ou seja, o DNA do zigoto é proveniente apenas do progenitor masculino o que pode ocorrer como resultado da fecundação de um óvulo sem cromossomos no núcleo (Figura 1), sendo referida como caso de partenogênese androgenética (CARLI; PEREIRA, 2017)
Figura 1 - Esquema de formação de mola hidatiforme completa (A) e parcial (B) respectivamente. (A) Espermatozoide normal fecunda ovócito sem material genético, em seguida, ocorre duplicação dos cromossomos advindos do gameta masculino que resulta em zigoto 46, XX com dissomia uniparental paterna que evolui para estado de total transformação em vesículas, denominado MHC. (B) Ocorrência de dispermia em ovócito normal resulta em zigoto triploide, em sequência, o trofoblasto é convertido em vesículas, porém, observa-se presença fetal.

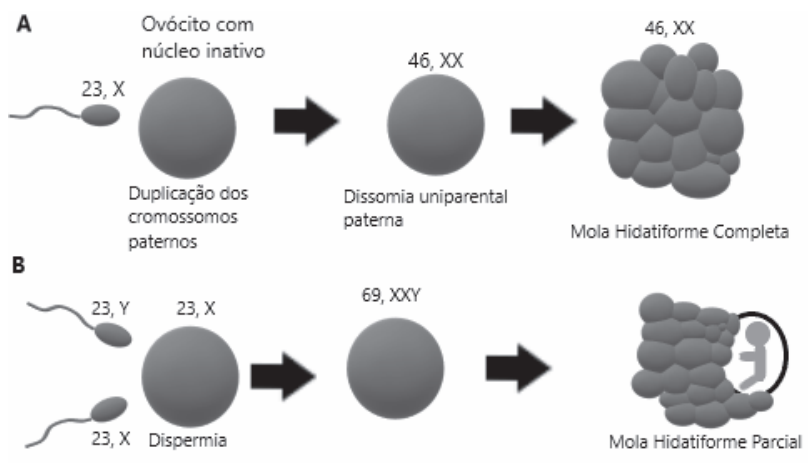

Fonte: Autoria própria

O presente estudo tem o objetivo de relacionar dificuldades reprodutivas com a presença de alterações cromossômicas na população atendida pelo programa Genética\&Sociedade do Instituto de Biologia da Universidade Federal da Bahia (UFBA), serviço comunitário que atende o público em geral e grupos específicos, constando de pessoas com deficiências e indígenas, encaminhados ao programa por centros públicos e filantrópicos.

\section{METODOLOGIA}

Estudo seccional descritivo baseado na análise de prontuários preenchidos durante aconselhamento genético de casais, com problemas reprodutivos, atendidos pelo programa supracitado. $O$ trabalho foi realizado durante o ano de 2018 recuperando informações acumuladas nos últimos 20 anos.

A análise das informações coletadas seguiu o seguinte protocolo: triagem, para selecionar entre o total de casais atendidos, aqueles que apresentassem dificuldades reprodutivas recorrentes; correlação cariótipo fenótipo, para verificar a possível associação entre a história de insucessos reprodutivos e alterações citogenéticas. Em seguida, selecionou-se os casais com alterações confirmadas para estudá-los com ênfase caso a caso. Tal grupo foi dividido em três categorias de acordo com a demanda que apresentaram: abortos recorrentes, molas hidatiformes e infertilidade. Posteriormente, as respectivas porcentagens foram calculadas e comparadas aos dados da literatura possibilitando verificar as semelhanças do público estudado com a população geral.

A técnica utilizada para investigar a constituição cromossômica dos indivíduos foi análise citogenética convencional com bandeamento GTG (MCGOWAN-JORDAN; SIMONS; SCHMID, 2016). A confecção dos 
gráficos e tabelas foi realizado com o auxílio do programa estatístico SPSS (IBM Statistics version 22.0).

$O$ presente artigo faz parte do projeto de pesquisa "Estudo longitudinal e correlação genótipo/fenótipo em indivíduos atendidos em serviço universitário de genética", submetido ao Comitê de Ética da Escola de Enfermagem da UFBA, aprovado de acordo com o Parecer no 1.023.774 de 01/04/2015, conforme a Resolução 466/12 do Conselho Nacional de Saúde.

\section{RESULTADOS}

Do total de 87 casais que foram atendidos no programa, 73 foram selecionados para esse estudo por buscarem aconselhamento genético devido a adversidades reprodutivas. Assim, essa motivação totalizou $83,9 \%$ de demanda de casais. Para os demais, o motivo da busca esteve centrado na ocorrência de filhos sindrômicos, com malformações ou outras condições genéticas na prole.

Entre os selecionados para este estudo, $59(80,8 \%)$ relataram abortos recorrentes, $9(12,3 \%)$ tinham histórico de molas hidatiformes e $5(6,8 \%)$ apresentaram infertilidade (Tabela 1). As alterações cromossômicas verificadas no grupo foram: translocações, inversões, trissomia de cromossomos sexuais e polimorfismos de regiões heterocromáticos. Os polimorfismos, não foram considerados por representarem variações sem consequências fenotípicas.

Tabela 1 - Correlação simplificada entre o número de casais com problemas reprodutivos, as respectivas condições $e$ porcentagens em relação ao total.

\begin{tabular}{llc}
\hline Casais & \multicolumn{1}{c}{ Condição } & Porcentagem \\
\hline 59 & Abortos recorrentes & $80,0 \%$ \\
09 & Mola Hidatiforme & $12,3 \%$ \\
05 & Infertilidade & $6,8 \%$ \\
\hline
\end{tabular}

Fonte: Dados da pesquisa

Após a análise dos históricos e dos resultados dos cariótipos, foi verificada a correlação com a alteração genética em somente 7 dos 73 casais, totalizando aproximadamente $9,59 \%$ do grupo estudado. Entre estes, 4 apresentaram translocações, 2 inversões e 1 mosaico da trissomia do cromossomo $X$. Vale ressaltar que em nenhum dos casos foi encontrada alteração citogenética nos dois parceiros. Nenhum caso de Mola Hidatiforme foi correlacionado a alterações citogenéticas.

\section{DISCUSSÃO}

Este estudo viabilizou reconhecer as taxas de danos reprodutivos associadas a anormalidades cromossômicas no público atendido no programa de genética comunitária bem como, verificar as características presentes nos históricos dos casais.

Entre os casais selecionados, alterações cromossômicas foram verificadas em aproximadamente 9,59\% dos casos. Em 80,8\% dos casos houve ocorrência de abortos repetitivos que representou a maior motivação para a busca por aconselhamento genético e também a maior taxa de achados citogenéticos anômalos. Estima-se que em $3 \%$ a $7 \%$ dos casais com abortos repetitivos um dos parceiros apresente alteração (FRANCISCO et al., 2013), em comparação, neste estudo, encontrou-se aproximadamente $10,17 \%$, superando taxas encontradas na literatura.

Molas hidatiformes foram a segunda condição mais presente $(12,3 \%)$ nos casos estudados e em todos os relatos ambos os progenitores possuíam cariótipo normal, sugerindo que a inconformidade cromossômica, apresentava-se somente nos produtos de concepção que, neste estudo, não foram investigados. Todavia, quase metade dos casos com Molas foram acompanhadas de outras condições adversas ao longo do histórico dos parceiros como: prole anterior nascida com malformação, Síndrome de Porter ou reincidência da $\mathrm{MH}$.

A infertilidade representou a motivação menos comum $(6,8 \%)$ entre os casais. Dentre os 7 cariótipos alterados apenas um estava relacionado à infertilidade e pertencia a progenitor masculino com inversão no cromossomo $\mathrm{Y}$.

Entre os casos de translocação, verificou-se a ocorrência de rearranjo raro 46,XX,t(1:20) (q42; q13), ou seja, translocação entre os cromossomos 1 e 20 no progenitor feminino caracterizada por histórico de 7 abortos entre 0 $3^{\circ}$ e $4^{\circ}$ mês de gestação, eventos que na literatura estão em acordo com a associação das translocações com o aumento da chance de abortos no primeiro semestre e do risco de perda gestacional espontânea. Esta translocação (Figura 2), tem sido relacionada a aborto no primeiro semestre, com risco de $50 \%$ de perda espontânea e baixa probabilidade de uma criança com a alteração não balanceada vir a nascer (MADAN; KLEINHOUT ,1987). 
Figura 2-Ideogramas esquemáticos de acordo com o ISCN (2016). (A) Da esquerda para direita cromossomos 1 e 20 normais respectivamente. (B) Translocação entre os cromossomos 1 e 20, respectivamente, com setas sinalizando os segmentos trocados entre os cromossomos.
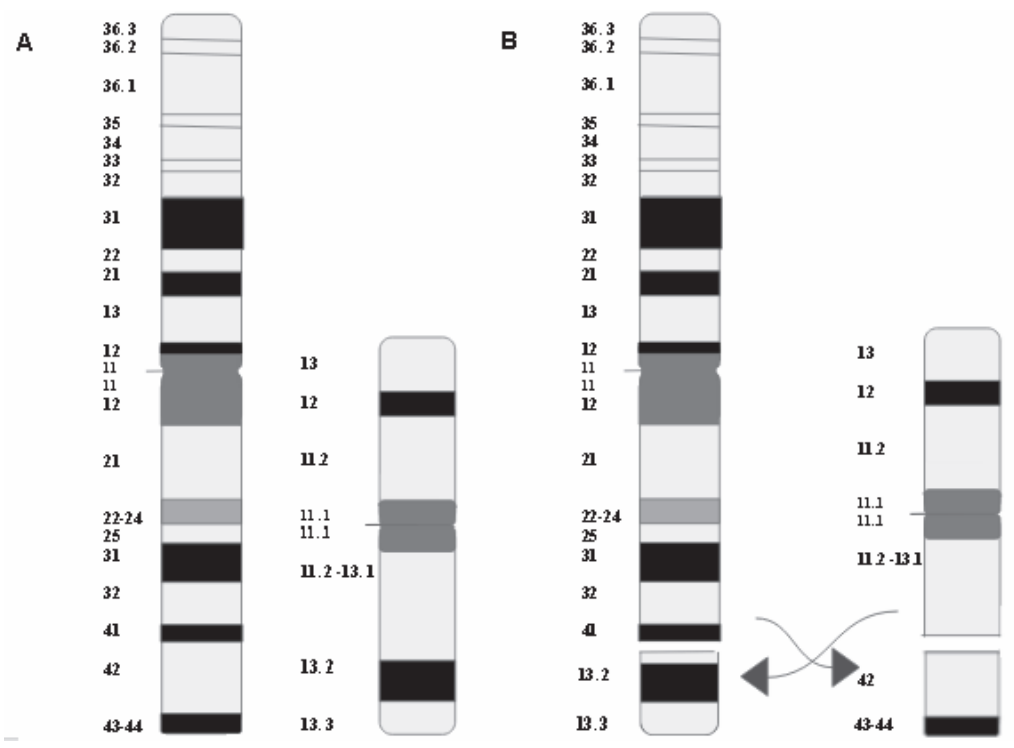

Fonte: Autoria própria

Outra condição investigada foi de paciente com mosaico 46,XX/47,XXX com histórico de três abortos precoces sempre no primeiro semestre. Um dos possíveis fatores do insucesso dessas gestações pode estar na falha de formação dos gametas no sistema reprodutivo materno. Os ovócitos de mulheres $46, X X / 47$, XXX estão em um estado de desenvolvimento abaixo do ideal e são propensos a letalidade embrionária (KUO; GUO, 2004).

Em um dos casais verificou-se cariótipo 46,XX,inv(14) (q31q32), ou seja, inversão paracêntrica balanceada no braço longo do cromossomo 14 do progenitor feminino. No histórico constavam quatro gestações bioquímicas, um óbito neonatal, um ovo anembrionado e ainda um aborto no primeiro trimestre. A paciente já estava na faixa de 40 anos, tornando complexo estimar a importância desse fator e da inversão nos acontecimentos sendo provável, porém, a presença de eventos de não-disjunção materna formando gametas cromossomicamente desbalanceados. Em Hassold e Sherman (2000) sugere-se a influência de dois possíveis eventos para essa não-disjunção. 0 primeiro, não vinculado a idade feminina, seria uma configuração vulnerável de quiasmas. $O$ segundo, vinculado a idade materna, estaria no processamento anormal de um bivalente vulnerável na meiose. Embora a não-disjunção possa ocorrer em qualquer faixa etária é mais comum com o aumento da idade, devido a degradação do processo meiótico que ocorre ao longo do tempo, logo é um fator a se considerar uma vez que a paciente apresentou êxito gestacional em gravidez anterior, em idade mais jovem. Observa-se que em contexto de inversões, como neste rearranjo, para maximizar o emparelhamento dos cromossomos homólogos na meiose, os mesmos se dobram sobre si formando um laço que permite aproximar as partes correspondentes dos cromossomos, a fim de que haja crossing-over entre os trechos correspondentes. Todavia, isto não garante que o processo ocorra de modo eficiente, podendo gerar gametas com problemas cromossômicos o que, consequentemente, poderia estar ligado aos fatos observados.

Três translocações robertsonianas foram identificadas: $\operatorname{rob}(13 ; 14), \operatorname{rob}(14 ; 21)$ e rob(15;21). Esses tipos de translocações ocorrem com a fusão centromérica entre dois cromossomos acrocêntricos. As mais comuns são as $\operatorname{rob}(13 ; 14)$ e rob(14;21), enquanto outras como $\operatorname{rob}(15 ; 21)$ e rob(13;15) são consideradas mais raras (FERFOURI et al., 2011). Todas estas translocações podem, entretanto, ocorrer em situações balanceadas originando indivíduos fenotipicamente normais (FERFOURI et al., 2011). Nos casos identificados nesse estudo que envolviam o cromossomo 21: rob(15;21) e rob(14;21), os quadros eram similares envolvendo perdas gestacionais e presença de síndrome de Down por translocação na trajetória familiar das respectivas probandas. Paralelamente, na probanda do caso com rob(13;14) foram registrados abortos, recém-nascido com a Síndrome de Patau (Trissomia do cromossomo 13) por translocação com morte precoce e finalmente uma criança normal sem o rearranjo.

As circunstâncias descritas estão associadas ao fato da translocação nos progenitores aumentarem as chances de formação de gametas desequilibrados (Figura 3). Após a fertilização, outras dificuldades como falha na implantação, abortos espontâneos, malformação congênita e nascimento de crianças com síndrome de Down (em translocações com o cromossomo 21) ou Síndrome de Patau (em translocações com o cromossomo 13) são resultados observados na literatura (FERFOURI et al., 2011). 
Figura 3 - Esquema demonstrando possíveis zigotos formados após fertilização de gametas provenientes de portador de translocação robertsoniana. (A) Célula germinativa portadora da translocação robertsoniana entre os cromossomos 14 e 21 (cor cinza e preta), do cromossomo 14 (somente cinza) e do cromossomo 21(somente preto) normais. (B) Possíveis configurações cromossômicas dos gametas formados. (C) Possíveis configurações cromossômicas de zigotos dos respectivos gametas após fertilização por gameta normal.

A

Célula germinativa com rob(14;21)

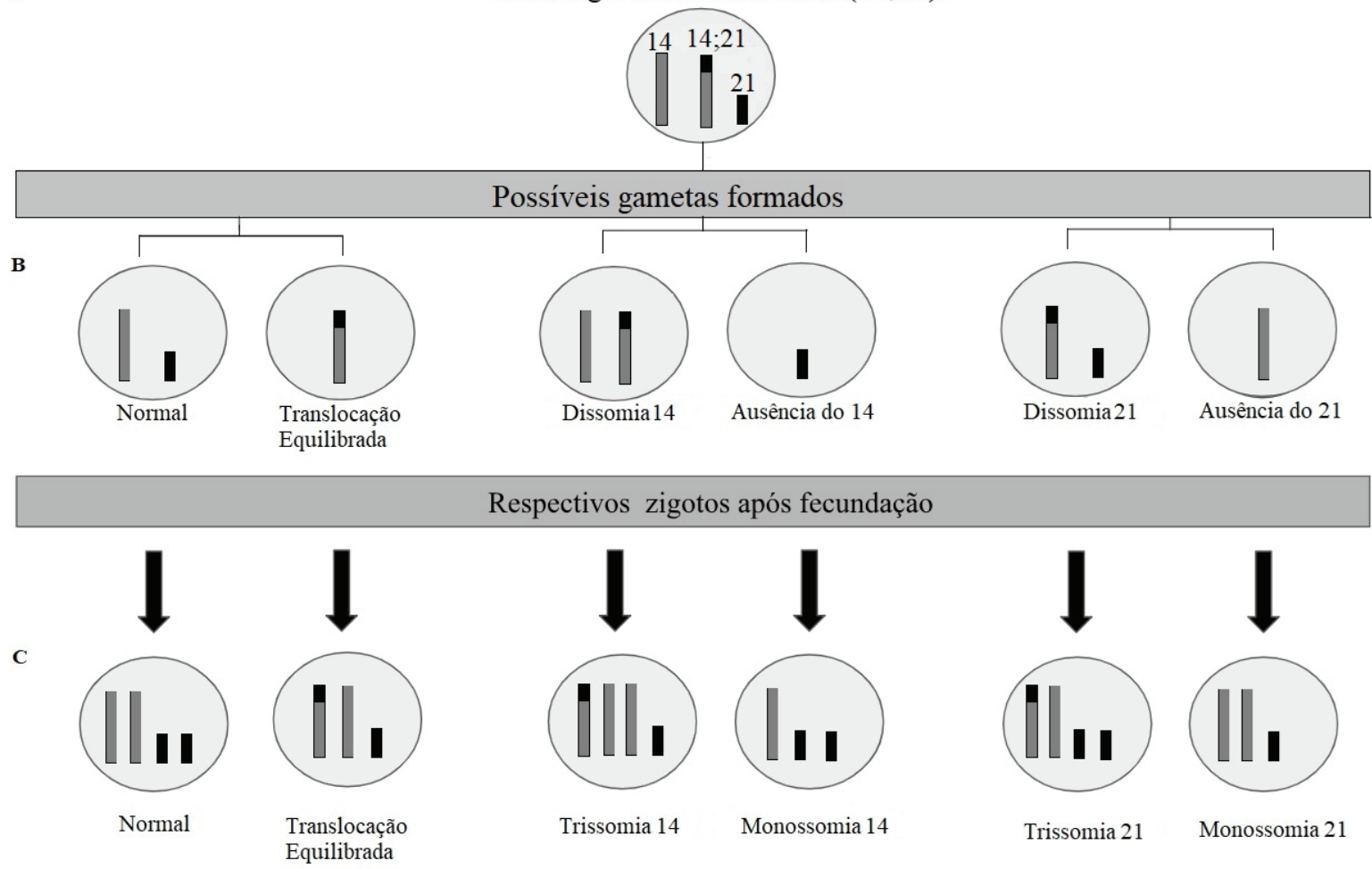

Fonte: Dados da pesquisa

Em relação ao exposto na Figura 3, deve ser ressaltado que algumas condições como as monossomias dos cromossomos 21 e 14 se apresentam letalmente ocasionando perda gestacional, enquanto a translocação equilibrada, por exemplo, poderá manter a alteração na genealogia.

De uma forma geral, as alterações cromossômicas encontradas interferem no processo reprodutivo. As mesmas podem ser condições novas, surgidas na prole, devido a eventos de não disjunção durante a formação dos gametas como ocorreu nos casos de mola hidatiforme relatadas, por exemplo. Bem como, podem estar ligadas a progenitores que tenham cariótipo anormal, aumentando as chances de formação de gametas com material cromossômico diferente do ideal acarretando em obstáculos para alcançar êxito da prole.

Considera-se ainda que apesar da fisiopatologia da perda gestacional devido a causas genéticas não ser clara, possa estar relacionada com uma implantação deficiente do embrião no útero pois o trofoblasto resultante de um cariótipo anormal parece não possuir uma capacidade adequada de invasão das artérias espiraladas maternas, de maneira a não permitir a evolução da gravidez no primeiro trimestre (FRANCISCO et al.,2013).

Os adultos presentes neste estudo apresentavam alterações balanceadas, consequentemente, não possuíam características divergentes da maioria das pessoas, por isso, para os mesmos, esse foi o primeiro aconselhamento genético do qual participaram. Semelhante ocorreu com os casais cujo cariótipo não diferiu do esperado.

\section{CONCLUSÃO}

O estudo realizado permitiu constatar que danos reprodutivos são responsáveis pelo achado de alterações cromossômicas em pessoas que, até então, jamais tiveram suspeita, visto que, eram portadores balanceados e que, portanto, as dificuldades reprodutivas constituem importante motivo de busca ao aconselhamento genético.

Embora as alterações citogenéticas não estivessem presentes na maioria dos casais com problemas gestacionais, ainda assim, se mostraram parte importante em meio a etiologia das dificuldades reprodutivas, já que, acarretaram em situações problemáticas recorrentes e em prejuízos pós-natal.

\section{REFERÊNCIAS}


ANDRADE, J. M. de. Mola hidatiforme e doença trofoblástica gestacional. Rev. bras. ginecol. obstet., Rio de Janeiro, v. 3, n. 2, p. 94-101, 2009.

CARLI, G. J. de; PEREIRA, T. C. On human parthenogenesis. Med. hypotheses, São Paulo, v. 106, p. 57-60, 2017.

DIBI, R. P.; PFEIL, J. N.; PASKULIN, G. A. Anormalidades cromossômicas em casais com história de aborto recorrente. Rev. bras. ginecol. obstet., Porto Alegre, v. 31, n. 2, p. 68-74, 2009.

EVSIKOV, S.; CIESLAK, J. M. L. T.; VERLINSKY, Y. Effect of chromosomal translocations on the development of preimplantation human embryos in vitro. Fertil. steril., Chicago, v. 74, n. 4, p. 672-677, 2000.

FERFOURI, F.; et al. The chromosomal risk in sperm from heterozygous Robertsonian translocation carriers is related to the sperm count and the translocation type. Fertil. steril., Chicago, v. 96, n. 6, p. 1337-1343, 2011.

FRANCISCO, C. et al.. Os factores genéticos na perda gestacional. Acta obstét. ginecol. port., p. 42-48, 2013.
HASSOLD, T.; SHERMAN, S. Down syndrome: genetic recombination and the origin of the extra chromosome 21. Clin. genet., Copenhagen, v. 57, n. 2 , p. $95-100,2000$.

MADAN, K.; KLEINHOUT, J. First trimester abortions associated with a translocation $t(1 ; 20)$ (p36; p11). Hum. genet., Berlin, v. 76, n. 1, p. 109-109,1987.

MARQUI, A. B. T. de. Anormalidades cromossômicas em abortos recorrentes por análise de cariótipo convencional. Rev. bras. saúde mater. infant., Recife, v. 18, n. 2, p. 277-288, 2018.

MCGOWAN-JORDAN, J.; SIMONS, A.; SCHMID, M. ISCN 2016: an international system for human cytogenomic nomenclature. Cytogenet. genome res., New York, v. 149, n. 1-2, 2016.

KUO, PAO-LIN; GUO, HOW-RAN. Mechanism of recurrent spontaneous abortions in women with mosaicism of X-chromosome aneuploidies. Fertil. steril., Taiwan, v. 82, n. 6, p. 1594-1601, 2004.

Submetido em: 08/04/2019

Aceito em: 08/11/2019 